\title{
Establishment of NOAEL for intracavernous injections of human bone marrow-derived mesenchymal stem cells in rats
}

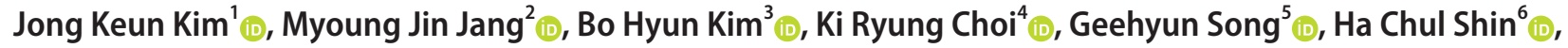

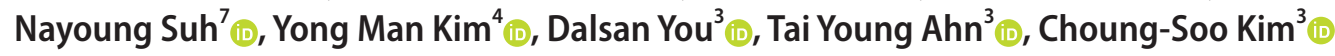 \\ ${ }^{1}$ Department of Urology, Hallym University Dongtan Sacred Heart Hospital, Hwaseong, ${ }^{2}$ Asan Institute for Life Science, Asan Medical Center, Seoul, ${ }^{3}$ Department of \\ Urology, Asan Medical Center, University of Ulsan College of Medicine, Seoul, ${ }^{4}$ NKMAX Co., Ltd., Seongnam, ${ }^{5}$ Department of Urology, Kangwon National University School \\ of Medicine, Chuncheon, ${ }^{6}$ Pharmicell Co., Ltd., Seongnam, 'Department of Pharmaceutical Engineering, College of Medical Sciences, Soon Chun Hyang University, Asan, \\ Korea
}

Purpose: To assess the possible negative health effects of human bone marrow-derived mesenchymal stem cells (hBMSCs) on fertility and early embryonic development following intracavernous injections in rats.

Materials and Methods: $A$ total of $88 \mathrm{Crl}: \mathrm{CD}(\mathrm{SD})$ male and female rats were equally divided into 4 groups in a random manner: control group (normal saline), low-dose group $\left(2 \times 10^{5} \mathrm{hBMSCs}\right)$, moderate-dose group $\left(1 \times 10^{6} \mathrm{hBMSCs}\right)$, and high-dose group $\left(2 \times 10^{6}\right.$ hBMSCs). hBMSCs or normal saline was injected into the penis of the rats 3 times at 2-week-intervals prior to mating. We compared each group with respect to parameters of reproduction and histopathology.

Results: For male rats, various degrees of flushing and swelling were observed at the penile injection site in all the groups, although the severity increased in a dose-dependent manner in the hBMSC injection groups. There were no statistically significant differences in mean body weights and food consumption among all the groups of both sexes. There were no statistically significant differences in reproductive parameters among all the groups of both sexes. The absolute and relative organ weights did not significantly differ among the groups. At the time of necropsy, no remarkable findings were observed in gross examinations in all groups. On histopathological analysis, minimal mononuclear cell infiltration was observed in the right epididymis of each rat in the moderate- and high-dose groups.

Conclusions: The non-toxic amount of hBMSCs for male fertility and early embryogenesis in rats under the test conditions was determined to be $2 \times 10^{6}$ cells/head.

Keywords: Embryonic development; Fertility; Rats; Stem cell transplantation; Toxic actions

This is an Open Access article distributed under the terms of the Creative Commons Attribution Non-Commercial License (http://creativecommons.org/licenses/by-nc/4.0) which permits unrestricted non-commercial use, distribution, and reproduction in any medium, provided the original work is properly cited.

\section{INTRODUCTION}

Erectile dysfunction (ED) is defined as the consistent or recurrent inability to attain and/or maintain penile erection sufficient for satisfactory sexual performance [1]. A large epidemiologic study showed that the overall prevalence of ED was $16 \%$ in men, and increased with age between 18 to 59 years. In another study, this prevalence was reported as

Received: 29 January, 2019 • Accepted: 21 August, 2019

Corresponding Author: Choung-Soo Kim (iD https://orcid.org/0000-0002-7464-3207

Department of Urology, Asan Medical Center, University of Ulsan College of Medicine, 88 Olympic-ro 43-gil, Songpa-gu, Seoul 05505, Korea

TEL: +82-2-3010-3734, FAX: +82-2-477-8928, E-mail: cskim@amc.seoul.kr 
$16.5 \%$ in men aged 18 to 29 years and $23.3 \%$ in men aged 50 to 59 years [2]. Phosphodiesterase type 5 (PDE5) inhibitors are commonly used to treat ED. However, an intact nitric oxide supply from the nerves and endothelium is required to ensure the efficacy of PDE5 inhibitors, which interfere with the nitric oxide-cyclic guanosine monophosphate pathway. Several prevalent diseases, including diabetes mellitus and denervation of the cavernous nerve due to pelvic surgeries, reduce the bioavailability of nitric oxide as a result of degeneration of the nitrinergic nerves supplying the penile corpora cavernosa and vasculature [3,4]. Therefore, the efficacy of PDE5 inhibitors in this population would not be expected to be as high as in the general population of ED patients [5].

Recent researches have shown that treatment with various stem cells could restore erectile function in preclinical models of cavernous nerve injury and diabetes mellitus [6]. Intracavernous stem cell delivery for $\mathrm{ED}$ treatment has been the most popular route due to the easy access and successful outcomes achieved thus far [7]. In a previous study, we noted that the intracavernous injection of human bone marrowderived mesenchymal stem cells (hBMSCs) led to the recovery of penile erection and histomorphometric changes in a rat model of cavernous nerve injury [8,9]. Several human clinical trials that used various stromal cells for ED treatment have been conducted [10-12], or are ongoing [6].

Infertility following hematopoietic cell transplantation occurs primarily because of the adverse effects of chemoradiotherapy [13]. In addition, acute and chronic graft-versushost disease (GVHD) may also be responsible for infertility after allogeneic hematopoietic cell transplantation [14,15]. However, there are few studies to report the reproductive and developmental influence of hBMSCs [16]. In the present study, we aimed to assess the possible negative health effects of hBMSCs on fertility and early embryonic development following intracavernous injections in rats.

\section{MATERIALS AND METHODS}

\section{Test material}

All the manufacturing and product testing procedures for the generation of hBMSCs were performed by Pharmicell Co. Ltd. (Seongnam, Korea). Approximately $10 \mathrm{~mL}$ of bone marrow was obtained from the posterior superior iliac crest of donor. Mononuclear cells were separated from the bone marrow and washed with phosphate-buffered saline (PBS). Cells were resuspended in Dulbecco's Modified Eagle's Medium-low glucose containing 10\% fetal bovine serum (Gibco; Thermo Fisher Scientific Inc., Waltham, MA, USA) and $20 \mu \mathrm{g} / \mathrm{mL}$ gentamicin and plated at a density of 10 to $1.5 \times 10^{5}$ cells $/ \mathrm{cm}^{2}$ in $75 \mathrm{~cm}^{2}$ or $175 \mathrm{~cm}^{2}$ flasks (Thermo Fisher Scientific Inc.). Cultures were maintained at $37^{\circ} \mathrm{C}$ in a humidified atmosphere containing $5 \% \mathrm{CO}_{2}$. After 5 to 7 days, the nonadherent cells were removed by replacing the medium and the adherent cells were cultured another 2 to 3 days. After reaching $70 \%$ to $80 \%$ confluence, the adherent cells were detached with trypsin containing ethylenediaminetetraacetic acid and replated at a density of 4 to $5 \times 10^{3}$ cells/ $\mathrm{cm}^{2}$ in $175 \mathrm{~cm}^{2}$ flasks. Cells were serially subcultured up to passage 5 for animal injection. On the day of injection, hBMSCs were harvested using trypsin, washed twice with PBS and once with Plasma Solution A Inj. (Multiple Electrolytes Injection, Type 1, USP; CJ HealthCare, Seoul, Korea) and resuspended to a final concentration of 1 to $4 \times 10^{7} \mathrm{cells} / \mathrm{mL}$ in Plasma Solution A Inj. Criteria for release of hBMSCs for preclinical use included absence of microbial contamination (bacteria, fungus, mycoplasma or endotoxin), viability greater than $70 \%$ when assessed using a trypan blue exclusion assay and immune phenotyping by flow cytometric analysis proving expression of CD73 and CD105 surface molecules (>85\%) and absence of CD14, CD34 and CD45 (<3\%) [9]. hBMSCs were provided from Pharmicell Co. Ltd. to Biotoxtech Co., Ltd. (Cheongju, Korea). Normal saline (JW Pharmaceutical, Seoul, Korea) was used as control material.

\section{Animal care}

The protocols for animal experimentation were approved by the Institutional Animal Care and Use Committee of Biotoxtech Co, Ltd. (130801), based on Korea's Animal Protection Act. A total of $95 \mathrm{Crl:CD(SD)} \mathrm{male} \mathrm{(6-week-old,}$ 163.0-190.5 g in weight) and female (9-week-old, 204.9-240.3 g in weight) rats were purchased from Orient Bio Inc. (Seongnam, Korea) and housed for 1 (for male rats) or 2 (for female rats) weeks for acclimatization. During the experiments, the rats were housed individually in stainless wire mesh cages $260 \times 350 \times 210 \mathrm{~mm}$ in size, and were maintained under a 12 hours:12 hours light/dark cycle (lights on at 07:00, lights off at $19: 00$ ), at a temperature of $20.4^{\circ} \mathrm{C}$ to $24.6^{\circ} \mathrm{C}$, and humidity of $35.9 \%$ to $68.7 \%$ with ad libitum access to food (Teklad Certified Global 18\% Protein Rodent Diet 2918C; Harlan Laboratories, Inc., Indianapolis, IN, USA) and water.

\section{Experimental design}

After acclimatization, 88 male (7-week-old, 237.9-277.6 g in weight) and female (11-week-old, 230.7-310.6 g in weight) rats, with body weight close to the mean value, were selected. The rats were equally divided in a random manner into 4 groups (22 male and female rats in each group), such 
that each group had similar mean body weight: (1) control group (normal saline), (2) low-dose group ( $\left.2 \times 10^{5} \mathrm{hBMSCs}\right)$, (3) moderate-dose group ( $\left.1 \times 10^{6} \mathrm{hBMSCs}\right)$, and (4) high-dose group $\left(2 \times 10^{6} \mathrm{hBMSCs}\right)$. Each male rat was anesthetized with Zoletil $50^{\circledR}$ (Tiletamin+Zolazepam; Virbac Laboratories, Carros, France). A $50 \mu \mathrm{L}$ suspension of hBMSCs (for the hBMSC injection groups) or normal saline (for the control group) was injected into the penis using a 30-gauge insulin syringe, 3 times at 2-week-intervals. The hBMSC doses and interval of injection were determined during a preliminary test conducted prior to the actual toxicity study, which included a preclinical dose-finding study for investigational new drug approval [9]. Female rats did not receive any treatment. The experimental design is outlined in Fig. 1.

\section{Observations of clinical signs}

All the rats were observed daily for general condition, motility, excreta, and other factors. Pregnant female rats were observed carefully for changes in the pregnancy state, such as abortion and premature birth. The presence of moribund conditions or mortality was also recorded.

\section{Body weight}

The body weights of male rats were measured twice a week from the dosing day to the necropsy day, whereas the body weights of female rats were measured twice a week from day 0 of gestation to the necropsy day.

\section{Food consumption}

The food consumption in male rats was measured weekly prior to dosing and during the observational period. After mating, the food consumption in female rats was measured weekly from day 1 of gestation to the necropsy day. The food consumption was not recorded during the mating period. The amount of food consumed was estimated by subtracting the amounts of leftover food from the amounts of presented

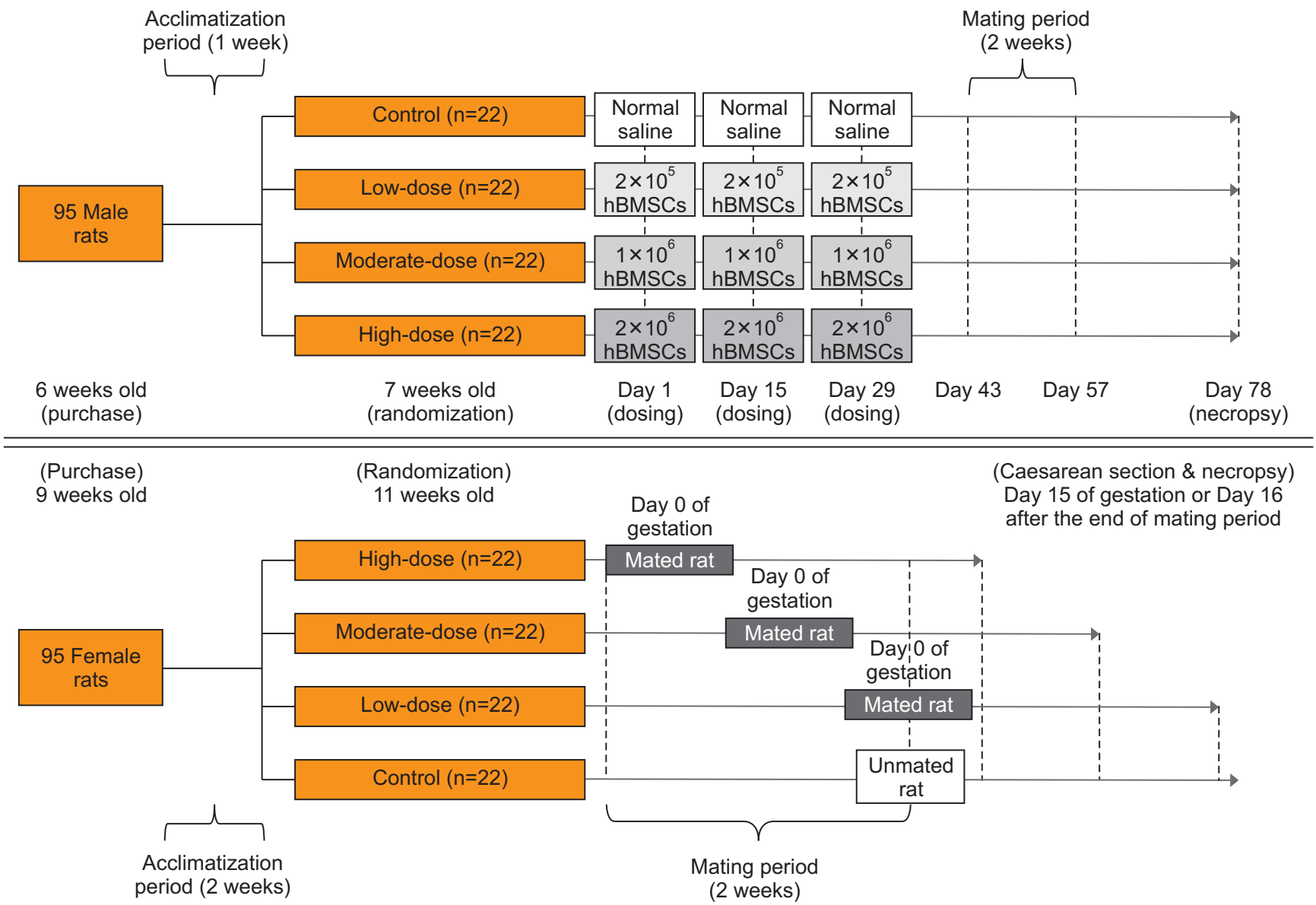

Fig. 1. Experimental design. For male rats, a $50 \mu \mathrm{L}$ suspension of human bone marrow-derived mesenchymal stem cells (hBMSCs) (for the hBMSC injection groups) or normal saline (for the control group) was injected into the penis, 3 times at 2-week-intervals. Male and female rats of the same group were cohabitated (1:1) for 2 weeks of the mating period, which began 2 weeks after the final injection in the male rats. Mating was evaluated based on the presence of a mating plug or via a vaginal smear test twice a day during the mating period. If mating was confirmed, the day was designated as day 0 of presumed gestation. Pregnancy was confirmed based on the presence of implantation in the uterus at the time of Caesarean section. All mated and unmated female rats underwent Caesarean section on day 15 of gestation or 16 days after the end of mating period, respectively. 
food.

\section{Mating}

Male rats cohabited with female rats of the same group (1:1) during the mating period. Mating was evaluated through the observation of a mating plug or via a vaginal smear test twice a day during the mating period. If mating was confirmed, the day was designated as day 0 of presumed gestation. Pregnancy was confirmed based on the presence of implantation in the uterus via a Caesarean section. Mating performance, including the mating index, fertility index, and pregnancy index, was calculated as described previously [17].

\section{Implantation rate and embryo mortality}

All mated and unmated female rats underwent a Caesarean section on day 15 of gestation or 16 days after the end of the mating period, respectively. The uteri were removed and the numbers of corpora lutea, implantation, and resorption were recorded. Implantation rate and embryo mortality were calculated as described previously [17]

\section{Necropsy}

Female rats were necropsied at the time of Caesarean section, and thereafter, the male rats were necropsied. All rats were sacrificed by exsanguination under isoflurane anesthesia, and the external surface and internal organs were then grossly examined.

\section{Organ weights}

At the time of necropsy in all male rats, the following organs were harvested and weighed individually: brain, pituitary, heart, liver, spleen, kidneys, adrenals, testes, and epididymides. The relative organ weights were calculated by dividing the absolute organ weights to the body weight.

\section{Histopathological analysis}

At the time of necropsy in all male rats, the following organs were harvested and fixed in 10\% neutral buffered formalin: brain, pituitary, heart, lung with bronchi, thyroid, liver, spleen, kidneys, adrenals, thymus, prostate, testes, epididymides, penis, seminal vesicles, and coagulating gland. Of these, the testes and epididymides were fixed in Bouin's solution first, followed by fixation in $10 \%$ neutral buffered formalin. The penis, left testis, and right epididymis of 10 male rats from each group were subjected to histopathological analysis.

\section{Sperm examinations}

Caudal epididymal sperm was examined in 10 male rats from each group at the time of necropsy. The left caudal epididymis was incised and incubated for approximately 3 to 10 minutes in $1 \%$ bovine serum albumin-Dulbecco's PBS. Samples were placed on glass slides and evaluated for motility by using a sperm analyzer (sperm analysis system, HTMTOX IVOS; Hamilton Thorne Biosciences, Beverly, MA, USA); the parameters of sperm motility were determined as described previously [17]. In addition, sperm samples from each group were smeared on glass slides, stained with DiffQuick, and examined for malformations using a light microscope at $200 \mathrm{sperms} / \mathrm{smear}$. The deformity rate was calculated as described previously [17].

Testicular sperm was examined in 10 male rats from each group at the time of necropsy. The right testis was
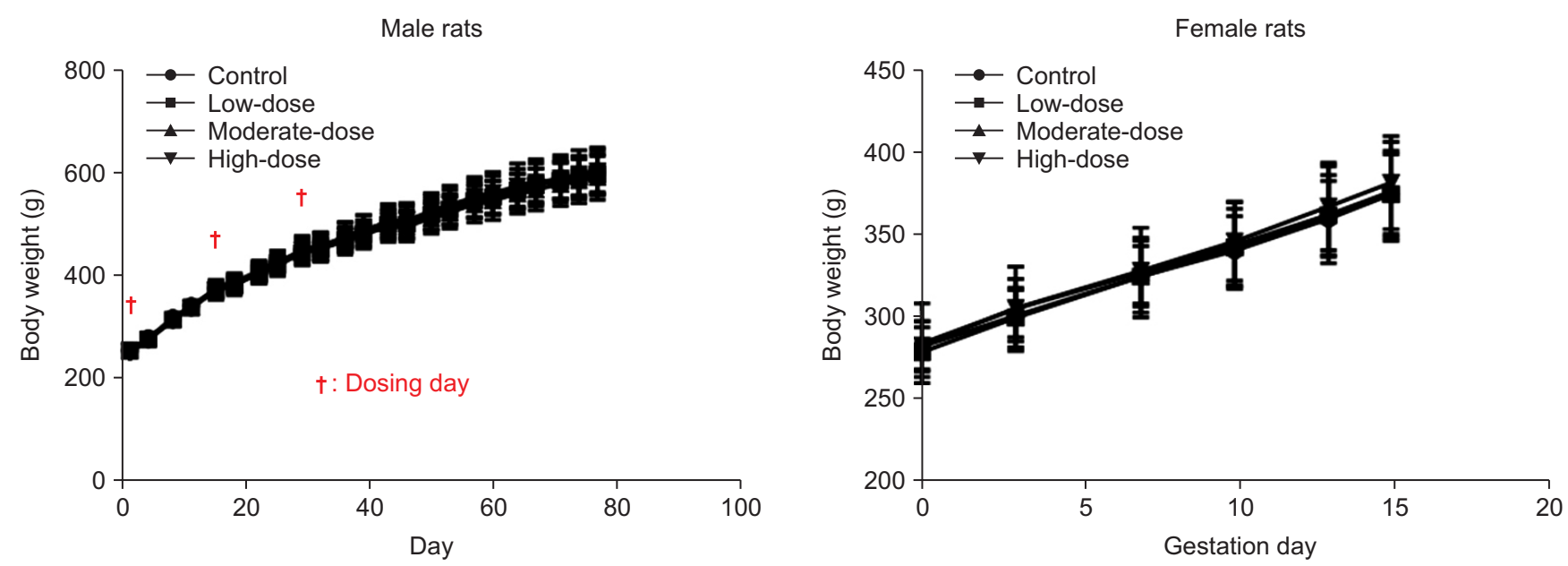

Fig. 2. Body weights of male and female rats. The body weight was analyzed using the Bartlett's test for homogeneity of variance. If equal variance was assumed, one-way analysis of variance was used, if significant, followed by the Dunnett's t-test for multiple comparisons. If equal variance was not assumed, the Kruskal-Wallis test was used, if significant, followed by the Steel's test for multiple comparisons. 
placed in $10 \mathrm{~mL}$ of distilled water, homogenized for $1 \mathrm{~min}$ utes using a homogenizer (Kinematica, Luzern, Switzerland), and sonicated for 3 minutes. One drop of the homogenization-resistant sperm heads solution was placed on the sperm counting chamber (makler counting chamber; Sefi Medical Instrument, Haifa, Israel) and the total sperm counts for each rat was determined using a microscope. The number of sperm per $1 \mathrm{~g}$ of right testis tissue was calculated.

\section{Statistical analysis}

Statistical significance was defined as $\mathrm{p}<0.05$ or $\mathrm{p}<0.01$. Statistical analysis was performed using SAS version 9.3 (SAS Institute Inc., Cary, NC, USA).
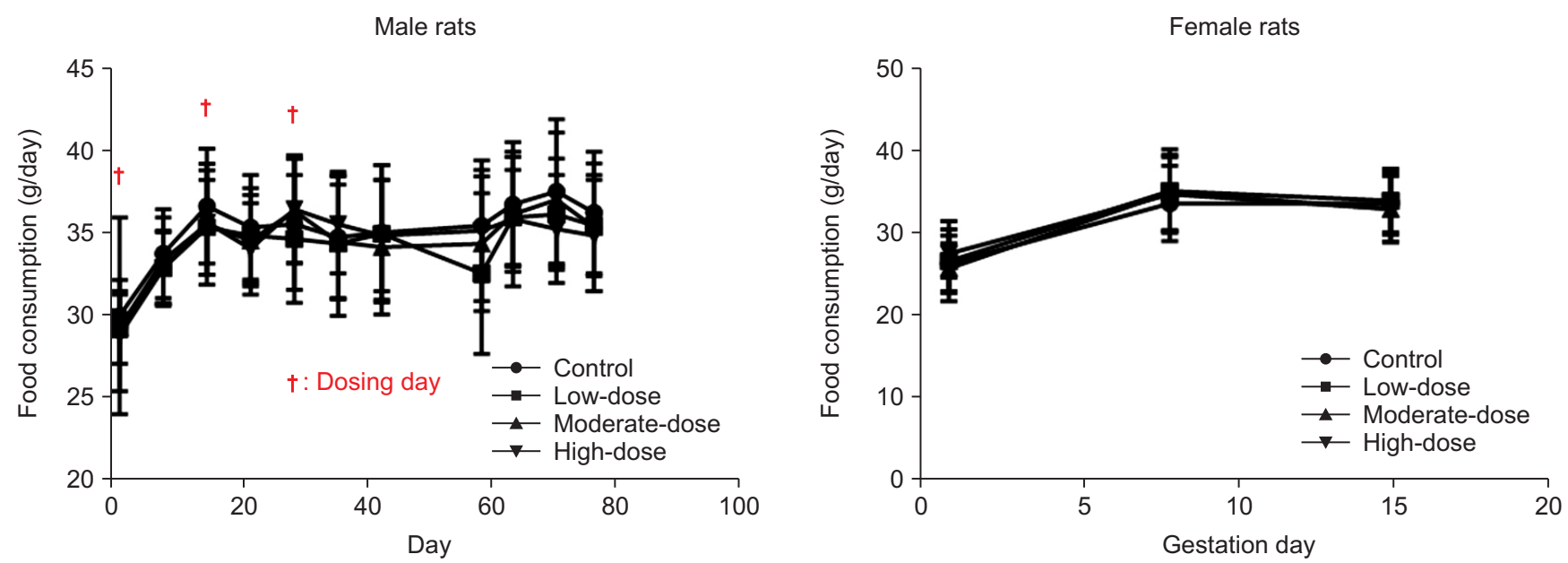

Fig. 3. Food consumption of male and female rats. The food consumption was analyzed using the Bartlett's test for homogeneity of variance. If equal variance was assumed, one-way analysis of variance was used, if significant, followed by the Dunnett's t-test for multiple comparisons. If equal variance was not assumed, the Kruskal-Wallis test was used, if significant, followed by the Steel's test for multiple comparisons.

Table 1. Summary of mating performance

\begin{tabular}{lccccrr}
\hline Group & $\begin{array}{c}\text { No. of female } \\
\text { rats placed with } \\
\text { male rats }\end{array}$ & $\begin{array}{c}\text { No. of } \\
\text { female rats } \\
\text { mated }\end{array}$ & $\begin{array}{c}\text { No. of } \\
\text { female rats } \\
\text { pregnant }\end{array}$ & $\begin{array}{c}\text { Mating } \\
\text { index (\%) }\end{array}$ & $\begin{array}{c}\text { Fertility } \\
\text { index (\%) }\end{array}$ & $\begin{array}{c}\text { Pregnancy } \\
\text { index (\%) }\end{array}$ \\
\hline Control & 22 & 21 & 20 & 95.5 & 95.2 & 90.9 \\
Low-dose & 22 & 21 & 19 & 95.5 & 90.5 & 86.4 \\
Moderate-dose & 22 & 22 & 22 & 100.0 & 100.0 & 100.0 \\
High-dose & 22 & 22 & 22 & 100.0 & 100.0 & 100.0 \\
\hline
\end{tabular}

Mating index $(\%)=($ No. of male or female rats mated/No. of female rats placed with male rats $) \times 100$

Fertility index $(\%)=($ No. of male or female rats fertilization/No. of mated male or female rats $) \times 100$

Pregnancy index $(\%)=($ No. of male or female rats pregnant/No. of female rats placed with male rats $) \times 100$

Mating performance was analyzed by Fisher's exact test.

Table 2. Summary of Caesarean section findings

\begin{tabular}{lccccc}
\hline \multicolumn{1}{c}{ Group } & No. of corpora lutea & No. of implantation & No. of resorption & Implantation rate (\%) & Embryo mortality (\%) \\
\hline Control $(n=20)$ & $18.1 \pm 3.5$ & $15.3 \pm 1.3$ & $0.2 \pm 0.4$ & $86.7 \pm 13.1$ & $1.3 \pm 2.6$ \\
Low-dose $(n=19)$ & $19.5 \pm 3.5$ & $15.3 \pm 2.2$ & $0.9 \pm 1.0$ & $79.6 \pm 13.7$ & $5.6 \pm 6.6$ \\
Moderate-dose $(n=22)$ & $19.3 \pm 4.7$ & $16.0 \pm 1.6$ & $0.8 \pm 0.8$ & $86.2 \pm 15.0$ & $4.9 \pm 5.2$ \\
High-dose $(n=22)$ & $20.8 \pm 4.2$ & $16.1 \pm 1.3$ & $0.5 \pm 0.9$ & $80.1 \pm 13.9$ & $3.3 \pm 5.1$ \\
\hline
\end{tabular}

Implantation rate $(\%)=($ No. of implantation/No. of corpora lutea $) \times 100$

Embryo mortality (\%)=(No. of resorption/No. of implantation) $\times 100$

Quantitative data were expressed as mean values with standard deviations.

The number of corpora lutea, the number of implantation, and implantation rate were analyzed using the Bartlett's test for homogeneity of variance. If equal variance was assumed, one-way analysis of variance was used, if significant, followed by the Dunnett's t-test for multiple comparisons. If equal variance was not assumed, the Kruskal-Wallis test was used, if significant, followed by the Steel's test for multiple comparisons. Embryo mortality was analyzed using the Kruskal-Wallis test was used, if significant, followed by the Steel's test for multiple comparisons. 


\section{RESULTS}

\section{Clinical signs}

No mortality case was observed in the control and hBMSC injection groups for both sex. For male rats, various degrees of flushing and swelling were observed at the penile injection site in the control and hBMSC injection groups. These features were more severe in the hBMSC injection groups than in the control group, and the severity increased in a dose-dependent manner. For female rats, no clinical signs were noted in the control or hBMSC injection groups (Supplementary Table 1).

\section{Body weight}

There were no statistically significant differences in the mean body weights among the control and hBMSC injection groups for both sexes (Fig. 2).

\section{Food consumption}

There were no statistically significant differences in the mean food consumption among the control and hBMSC injection groups for both sexes (Fig. 3).

\section{Mating}

There were no statistically significant differences in mating performance among the control and hBMSC injection groups (Table 1). In addition, the mean duration from inception of cohabitation to mating was similar in all the groups (2.4, 2.2, 2.9, and 2.1 days for the control, low-dose, moderate-dose, and high-dose groups, respectively).

\section{Implantation rate and embryo mortality}

At the time of Caesarean section, the implantation rates were found to be $86.7 \%, 79.6 \%, 86.2 \%$, and $80.1 \%$, whereas the embryo mortality rates were found to be $1.3 \%, 5.6 \%, 4.9 \%$, and $3.3 \%$ for the control, low-dose, moderate-dose, and highdose groups, respectively (Table 2). There were no significant differences in the implantation rate and embryo mortality among the control and hBMSC injection groups.

\section{Necropsy findings}

No remarkable findings were observed on gross examinations in any of the groups (Supplementary Table 2).

\section{Organ weights}

There were no significant differences in the absolute and relative organ weights among the control and hBMSC injection groups (Tables 3 and 4).

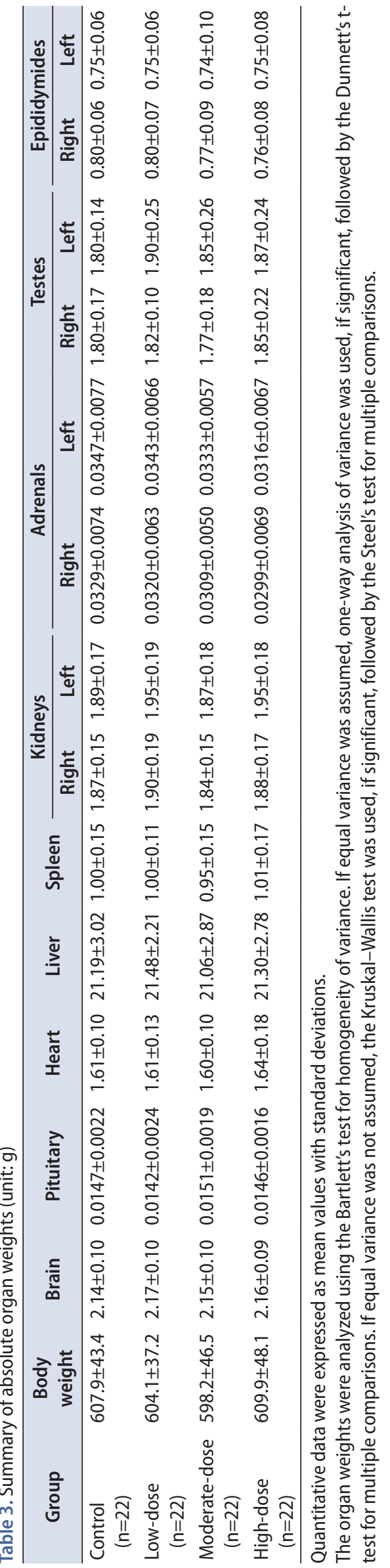




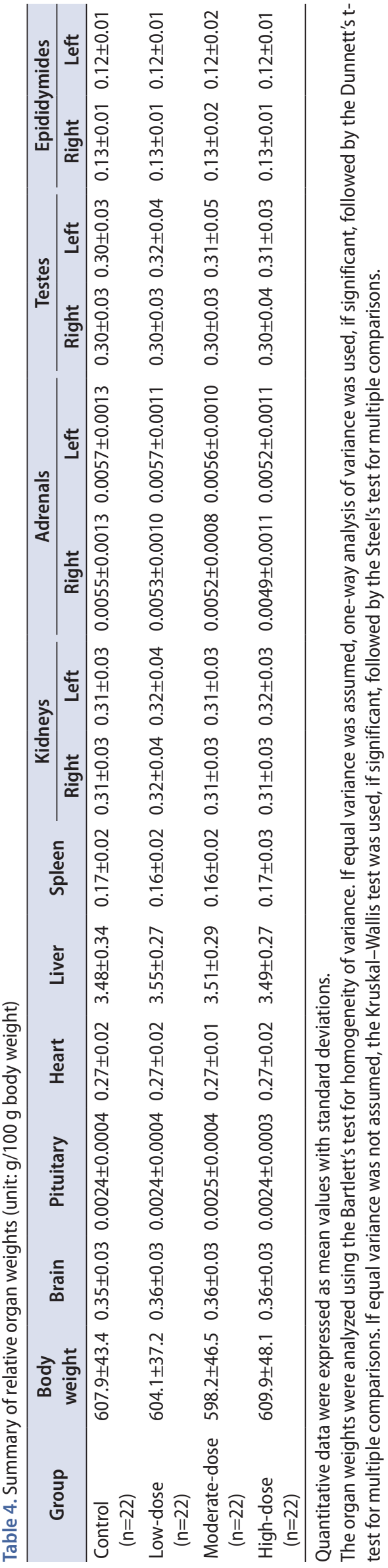

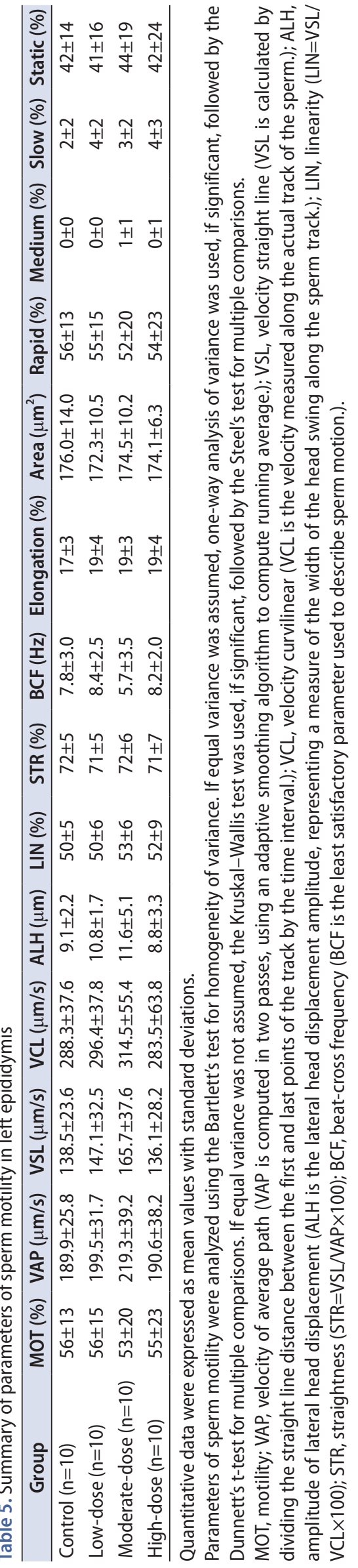

https://doi.org/10.4111/icu.2020.61.1.88 
Table 6. Summary of sperm morphology in left epididymis

\begin{tabular}{|c|c|c|c|c|c|c|c|}
\hline \multirow{3}{*}{ Group } & \multicolumn{6}{|c|}{ Abnormal sperm ratio (\%) } & \multirow{3}{*}{ Deformity (\%) } \\
\hline & \multicolumn{2}{|c|}{ Head } & \multirow{2}{*}{$\begin{array}{c}\text { Midpiece } \\
\text { Bent }\end{array}$} & \multicolumn{2}{|c|}{ Tail } & \multirow{2}{*}{$\begin{array}{l}\text { Head \& tail } \\
\text { separation }\end{array}$} & \\
\hline & Bent neck & Two & & Coil & Small & & \\
\hline Control $(n=10)$ & $0.2 \pm 0.3$ & $0.1 \pm 0.2$ & $0.6 \pm 0.4$ & $0.8 \pm 0.8$ & $0.3 \pm 0.4$ & $2.4 \pm 1.2$ & $4.2 \pm 1.9$ \\
\hline Low-dose $(n=10)$ & $0.2 \pm 0.4$ & $0.1 \pm 0.2$ & $0.3 \pm 0.5$ & $1.0 \pm 1.1$ & $0.2 \pm 0.3$ & $2.0 \pm 1.1$ & $3.7 \pm 2.0$ \\
\hline Moderate-dose $(n=10)$ & $0.2 \pm 0.3$ & $0.0 \pm 0.0$ & $0.3 \pm 0.4$ & $0.8 \pm 0.9$ & $0.3 \pm 0.5$ & $2.2 \pm 0.9$ & $3.6 \pm 1.9$ \\
\hline High-dose $(n=10)$ & $0.3 \pm 0.4$ & $0.1 \pm 0.2$ & $0.5 \pm 0.6$ & $0.7 \pm 0.9$ & $0.4 \pm 0.3$ & $2.3 \pm 1.4$ & $4.1 \pm 1.9$ \\
\hline
\end{tabular}

Quantitative data were expressed as mean values with standard deviations.

Sperm malformations were analyzed using the Bartlett's test for homogeneity of variance. If equal variance was assumed, one-way analysis of variance was used, if significant, followed by the Dunnett's t-test for multiple comparisons. If equal variance was not assumed, the Kruskal-Wallis test was used, if significant, followed by the Steel's test for multiple comparisons. Sperm deformity was analyzed using the Kruskal-Wallis test was used, if significant, followed by the Steel's test for multiple comparisons.

Table 7. Summary of sperm count in right testis

\begin{tabular}{lc}
\hline \multicolumn{1}{c}{ Group } & No. of sperms $\left(\times 10^{6} / \mathrm{g}\right)$ \\
\hline Control $(\mathrm{n}=10)$ & $191 \pm 27$ \\
Low-dose $(\mathrm{n}=10)$ & $189 \pm 39$ \\
Moderate-dose $(\mathrm{n}=10)$ & $196 \pm 38$ \\
High-dose $(\mathrm{n}=10)$ & $201 \pm 39$ \\
\hline
\end{tabular}

Quantitative data were expressed as mean values with standard deviations.

Sperm count was analyzed using the Bartlett's test for homogeneity of variance. If equal variance was assumed, one-way analysis of variance was used, if significant, followed by the Dunnett's t-test for multiple comparisons. If equal variance was not assumed, the Kruskal-Wallis test was used, if significant, followed by the Steel's test for multiple comparisons.

\section{Histopathological analysis}

Even though minimal mononuclear cell infiltration in right epididymis was observed in each a rat of the moderateand high-dose groups, no remarkable findings were observed on histopathological analysis in any of the groups (Supplementary Table 3).

\section{Sperm examinations}

There were no significant differences in sperm motility, malformation, and sperm count among the control and hBMSC injection groups (Tables 5-7).

\section{DISCUSSION}

Most pre-transplant conditioning regimens for hematopoietic cell transplantation include alkylating agents and/ or irradiation, both of which may cause germ cell injury, gonadal dysfunction, and infertility [13]. Both acute and chronic GVHD can lead to infertility after allogeneic hematopoietic cell transplantation, through a loss of Leydig cell function during a local alloresponse or via impaired spermatogenesis due to the effects of systemic inflamma- tory factors of ongoing GVHD [14,15]. Although hBMSCs are not inherently immunogenic due to the lack of major histocompatibility complex human leukocyte antigen class II [18] transplant rejection can occur in normal rats without immunosuppression [19]. In addition, repeated transplantation of human embryonic stromal cells into immunocompetent mice reportedly led to accelerated cell death due to an adaptive donor-specific immune response [9,18,20]. Therefore, hBMSCs might cause reproductive and developmental toxicity. In the present study, we aimed to assess the possible negative health effects of hBMSCs on fertility and early embryonic development following intracavernous injections in male rats before mating.

We did not observe any hBMSCs treatment-related deaths. Flushing and swelling at the penile injection site were observed in the control and hBMSC injection groups, wherein the severity increased in a dose-dependent manner. Hence, hBMSCs can be considered to have minor effects at the penile injection site. Nevertheless, this reaction does not appear to have any toxicological significance, as mating performance, pregnancy, or sperm remain unaffected. Over the entire course of the study, we did not observe any significant alterations in body weights or food consumption in any of the hBMSC-treated rats of both sexes.

Moreover, there were no significant hBMSC-induced negative health effects on any of the reproductive parameters in both sexes. There were also no significant gross or histopathological findings related to hBMSC-induced toxicity in both sexes. The minimal mononuclear cell infiltration in the right epididymis observed in each rat in the moderate- and high-dose groups was not associated with any corresponding reproductive manifestations. Both pairs of male/ female rats mated successfully, and pregnancy was noted. The implantation rate of each pair was $84.2 \%$ and $56.7 \%$, respectively, and no embryo mortality was noted in either pair.

Moreover, both pairs did not exhibit any noticeable de- 
viation in sperm motility, malformation, and sperm count fertility from the mean of each group. Therefore, these histopathological changes were not considered to be associated with hBMSC-related adverse effects, but appeared to be adventitious due to individual differences.

There are several limitations in the present study. The modulation of paracrine factors such as exosome and mitochondrial transfer for tissue repair were important in other organs [21]. But, our study had to suggest a direct toxicity of stem cell treatment at ED rats. Although we could not show the paracrine effects of hBMSC related with infertility and early embryonal development, we considered that this could affect the infertility and early embryogenesis. Thus, we continue to observe and work out this effect in subsequent studies. Also, we could not exclude immune rejection. The macrophage-mediated immune response in the injection site increased significantly after injection of stem cells [19]. Our previous study revealed that grade of inflammation was significantly increased in the delayed multiple hBMSCs injection rats. In addition, some inflammation was observed in the control, immediate hBMSCs injection, and delayed single hBMSCs injection groups [9]. This might be attributable to trauma by needle penetration or weak xenogenic reaction. We suggest that multiple hBMSCs injections induce an immune response that justifies the absence of incremental benefit of repeated stem cell treatments, as confirmed by the presence of inflammation. We proposed to assess the possible negative health effects of hBMSCs on fertility and early embryonic development following intracavernous injections in male rats before mating. Flushing and swelling at the penile injection site were observed in the control and $\mathrm{hBMSC}$ injection groups, wherein the severity increased in a dose-dependent manner. Hence, hBMSCs can be considered to have minor effects at the penile injection site. Nevertheless, this reaction does not appear to have any toxicological significance, as mating performance, pregnancy, or sperm remain unaffected.

To our knowledge, this study is the first to evaluate the possible negative effects of stem cell therapy on fertility and early embryonic development. We have been performing a phase I study to evaluate the safety of autologous BMSCs in $\mathrm{ED}$, based on our current results and those of a previous report on efficacy outcomes $[9,22]$. Furthermore, the purpose of this study is to present the basis for the safe use of hBMSCs in ED patients.

\section{CONCLUSIONS}

This study assessed the potential adverse effects of
hBMSCs injected into the penis of male SD rats, 3 times at 2-week-intervals, prior to mating. There were no significant changes in body weight, food consumption, organ weight, necropsy, and reproductive parameters of both sexes, except for a minor local effect at the penile injection site and an unrelated histopathological change in the right epididymis. Therefore, a non-toxic amount for male fertility and early embryogenesis of rats under the test conditions (NOAEL) was determined to be $2 \times 10^{6}$ cells/head.

\section{CONFLICTS OF INTEREST}

The authors have nothing to disclose.

\section{ACKNOWLEDGMENTS}

This study was supported by a grant of the Korean Health Technology R\&D Project, Ministry of Health and Welfare, Republic of Korea (A121957). The funders had no role in study design, data collection and analysis, decision to publish, or preparation of the manuscript.

The authors thank the participating investigators at Biotoxtech Co., Ltd. for the performance of experimental tasks and/or collection of data.

\section{AUTHORS' CONTRIBUTIONS}

Research conception and design: Choung-Soo Kim, Dalsan You and Yong Man Kim. Performing the experiments: Jong Keun Kim, Myoung Jin Jang, Ki Ryung Choi, Geehyun Song, Bo Hyun Kim, Ha Chul Shin and Dalsan You. Data analysis and interpretation: Jong Keun Kim, Ki Ryung Choi, Geehyun Song, Ha Chul Shin, Nayoung Suh and ChoungSoo Kim. Statistical analysis: Jong Keun Kim, Ki Ryung Choi, Geehyun Song, Ha Chul Shin and Dalsan You. Drafting of the manuscript: Jong Keun Kim, Myoung Jin Jang, Bo Hyun Kim, Dalsan You and Choung-Soo Kim. Critical revision of the manuscript Jong Keun Kim, Dalsan You and Choung-Soo Kim. Supervision other (specify): Nayoung Suh, Tai Young Ahn and Choung-Soo Kim. Receiving grant: Choung-Soo Kim. Approval of final manuscript: all authors.

\section{SUPPLEMENTARY MATERIALS}

Scan this QR code to see the supplementary materials, or visit https://www.icurology.org/src/sm/icurology-61-88-s001.pdf. 


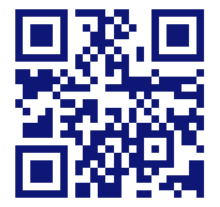

\section{REFERENCES}

1. McCabe MP, Sharlip ID, Atalla E, Balon R, Fisher AD, Laumann E, et al. Definitions of sexual dysfunctions in women and men: a consensus statement from the fourth International Consultation on Sexual Medicine 2015. J Sex Med 2016;13:13543.

2. Goldstein I, Chambers R, Tang W, Stecher V, Hassan T. Realworld observational results from a database of 48 million men in the United States: relationship of cardiovascular disease, diabetes mellitus and depression with age and erectile dysfunction. Int J Clin Pract 2018;72:e13078.

3. Cellek S, Rodrigo J, Lobos E, Fernández P, Serrano J, Moncada $\mathrm{S}$. Selective nitrergic neurodegeneration in diabetes mellitusa nitric oxide-dependent phenomenon. Br J Pharmacol 1999;128:1804-12.

4. Ozkara H, Alan C, Atukeren P, Uyaner I, Demirci C, Gümüştaş $\mathrm{MK}$, et al. Changes of nitric oxide synthase-containing nerve fibers and parameters for oxidative stress after unilateral cavernous nerve resection or manuplation in rat penis. Chin J Physiol 2006;49:160-6.

5. Suetomi T, Kawai K, Hinotsu S, Joraku A, Oikawa T, Sekido N, et al. Negative impact of metabolic syndrome on the responsiveness to sildenafil in Japanese men. J Sex Med 2008;5:144350.

6. Soebadi MA, Moris L, Castiglione F, Weyne E, Albersen M. Advances in stem cell research for the treatment of male sexual dysfunctions. Curr Opin Urol 2016;26:129-39.

7. Alwaal A, Hussein AA, Lin CS, Lue TF. Prospects of stem cell treatment in benign urological diseases. Korean J Urol 2015;56:257-65.

8. You D, Jang MJ, Lee J, Jeong IG, Kim HS, Moon KH, et al. Periprostatic implantation of human bone marrow-derived mesenchymal stem cells potentiates recovery of erectile function by intracavernosal injection in a rat model of cavernous nerve injury. Urology 2013;81:104-10.

9. You D, Jang MJ, Kim BH, Choi KR, Lee C, Song G, et al. Bone marrow-derived mesenchymal stromal cell therapy in a rat model of cavernous nerve injury: preclinical study for approval. Cytotherapy 2016;18:870-80.

10. Bahk JY, Jung JH, Han H, Min SK, Lee YS. Treatment of diabetic impotence with umbilical cord blood stem cell intracavernosal transplant: preliminary report of 7 cases. Exp Clin
Transplant 2010;8:150-60.

11. Yiou R, Hamidou L, Birebent B, Bitari D, Lecorvoisier P, Contremoulins I, et al. Safety of intracavernous bone marrowmononuclear cells for postradical prostatectomy erectile dysfunction: an open dose-escalation pilot study. Eur Urol 2016;69:988-91.

12. Haahr MK, Jensen CH, Toyserkani NM, Andersen DC, Damkier P, Sørensen JA, et al. Safety and potential effect of a single intracavernous injection of autologous adipose-derived regenerative cells in patients with erectile dysfunction following radical prostatectomy: an open-label phase I clinical trial. EBioMedicine 2016;5:204-10.

13. Carter A, Robison LL, Francisco L, Smith D, Grant M, Baker KS, et al. Prevalence of conception and pregnancy outcomes after hematopoietic cell transplantation: report from the Bone Marrow Transplant Survivor Study. Bone Marrow Transplant 2006;37:1023-9.

14. Wagner AM, Beier K, Christen E, Holländer GA, Krenger W. Leydig cell injury as a consequence of an acute graft-versushost reaction. Blood 2005; 105:2988-90.

15. Rovó A, Aljurf M, Chiodi S, Spinelli S, Salooja N, Sucak G, et al.; Late Effects Working Party of the EBMT. Ongoing graftversus-host disease is a risk factor for azoospermia after allogeneic hematopoietic stem cell transplantation: a survey of the Late Effects Working Party of the European Group for Blood and Marrow Transplantation. Haematologica 2013;98:339-45.

16. Rengasamy M, Gupta PK, Kolkundkar U, Singh G, Balasubramanian S, SundarRaj S, et al. Preclinical safety \& toxicity evaluation of pooled, allogeneic human bone marrow-derived mesenchymal stromal cells. Indian J Med Res 2016;144:852-64.

17. Yimam M, Lee YC, Hyun EJ, Jia Q. Reproductive and developmental toxicity of orally administered botanical composition, UP446-part III: effects on fertility and early embryonic development to implantation in Sprague Dawley rats. Birth Defects Res B Dev Reprod Toxicol 2015;104:166-76.

18. Le Blanc K, Tammik C, Rosendahl K, Zetterberg E, Ringdén O. HLA expression and immunologic properties of differentiated and undifferentiated mesenchymal stem cells. Exp Hematol 2003;31:890-6.

19. Grinnemo KH, Månsson A, Dellgren G, Klingberg D, Wardell E, Drvota V, et al. Xenoreactivity and engraftment of human mesenchymal stem cells transplanted into infarcted rat myocardium. J Thorac Cardiovasc Surg 2004;127:1293-300.

20. Auchincloss H Jr, Sachs DH. Xenogeneic transplantation. Annu Rev Immunol 1998;16:433-70.

21. Liang X, Ding Y, Zhang Y, Tse HF, Lian Q. Paracrine mechanisms of mesenchymal stem cell-based therapy: current status and perspectives. Cell Transplant 2014;23:1045-59. 
22. ClinicalTrials.gov. Safety of autologous bone marrow derived mesenchymal stem cells in erectile dysfunction [Internet]. Bethesda: U.S. National Library of Medicine; 2015 Jan 26 [(up- dated 2019 Jan 9;) cited 2016 Jul 31]. Available from: https:// www.clinicaltrials.gov/ct2/show/NCT02344849. 